\title{
ON INTERPOLATION BY ALMOST TRIGONOMETRIC SPLINES ${ }^{1}$
}

\author{
Sergey I. Novikov \\ Krasovskii Institute of Mathematics and Mechanics, \\ Ural Branch of the Russian Academy of Sciences, \\ Ekaterinburg, Russia \\ Sergey.Novikov@imm.uran.ru
}

\begin{abstract}
The existence and uniqueness of an interpolating periodic spline defined on an equidistant mesh by the linear differential operator $\mathcal{L}_{2 n+2}(D)=D^{2}\left(D^{2}+1^{2}\right)\left(D^{2}+2^{2}\right) \cdots\left(D^{2}+n^{2}\right)$ with $n \in \mathbb{N}$ are reproved under the final restriction on the step of the mesh. Under the same restriction, sharp estimates of the error of approximation by such interpolating periodic splines are obtained.
\end{abstract}

Key words: Splines, Interpolation, Approximation, Linear differential operator.

\section{Introduction}

Let $D=d / d x, n \in \mathbb{N}$, and let

$$
\mathcal{L}_{2 n+2}(D)=D^{2}\left(D^{2}+1^{2}\right)\left(D^{2}+2^{2}\right) \cdots\left(D^{2}+n^{2}\right)
$$

be the $(2 n+2)$ th-order linear differential operator with constant real coefficients. We denote the characteristic polynomial of $\mathcal{L}_{2 n+2}(D)$ by $p_{2 n+2}$, and let $T_{2 n+2}=\{0,0, \pm i, \ldots, \pm i n\}$ be the set of its zeros, with each zero repeated according to its multiplicity, where $i$ is the imaginary unit. The kernel of the differential operator $(0.1)$ is the linear space spanned by the system of functions $\{1, x, \sin x, \cos x, \ldots, \sin n x, \cos n x\}$.

Denote by $\mathbb{T}$ the circumference considered as the interval $[0,2 \pi]$ with identified ends, and let $\|\cdot\|_{L_{p}(\mathbb{T})}=\|\cdot\|_{p} \quad(1 \leq p \leq \infty)$ with the usual modification in the case $p=\infty$.

We associate with the differential operator $\mathcal{L}_{2 n+2}(D)$ the standard class of differentiable functions

$$
W_{\infty}\left(\mathcal{L}_{2 n+2}\right)=\left\{f \in C^{(2 n+1)}(\mathbb{T}): f^{(2 n+1)} \text { is abs. cont., }\left\|\mathcal{L}_{2 n+2}(D) f\right\|_{\infty} \leq 1\right\} .
$$

Let $N \in \mathbb{N}$ and $h=\pi / N$. Denote by $\Delta_{N}=\{j h: j=0,1, \ldots, 2 N-1\}$ the uniform mesh on $[0,2 \pi)$ which can be extended on $\mathbb{R}$ if required; $h$ is the step of the mesh.

We say that a $2 \pi$-periodic function $s_{2 n+2}$ is a periodic almost trigonometric spline with knots at the points of $\Delta_{N}$ if $s_{2 n+2}$ satisfies the following conditions:

1) $s_{2 n+2} \in C^{(2 n)}(\mathbb{T})$,

2) $\mathcal{L}_{2 n+2}(D) s_{2 n+2}(x)=0 \quad \forall x \in(j h,(j+1) h), \quad j \in \mathbb{Z}$.

The set of all almost trigonometric splines is denoted by $S\left(\mathcal{L}_{2 n+2}, \Delta_{N}\right)$.

Almost trigonometric splines are a special case of the large family of $\mathcal{L}$-splines defined by linear differential operators (see [2], [3], [8], and others).

The term "almost trigonometric spline" is explained by the fact that such a spline is formed by functions which differ from trigonometric polynomials for only one addend $a x$, where $a$ is some

\footnotetext{
${ }^{1}$ This work was supported by the Program "Modern problems in function theory and applications" of the Ural Branch of RAS (project no. 15-16-1-4).
} 
constant. This term is not standard, and we use it only not to specify every time by what differential operator the considered splines are defined.

We interpolate at the knots of the mesh $\Delta_{N}$ by elements from $S\left(\mathcal{L}_{2 n+2}, \Delta_{N}\right)$; i.e., for every bounded $2 N$-periodic sequence $y=\left\{y_{\nu}: \nu \in \mathbb{Z}\right\}, y_{\nu}=y_{\nu+2 N}$, we consider the interpolation problem: to find $s \in S\left(\mathcal{L}_{2 n+2}, \Delta_{N}\right)$ such that $s(\nu h)=y_{\nu}, \nu \in \mathbb{Z}$.

For interpolation by polynomial splines, the existence, uniqueness and estimates of the error of approximation in many classes of functions are well-known (see, for instance, [1, Ch. V], [11], [12], [13], and references therein).

The existence and uniqueness of periodic interpolating $L$-splines corresponding to an arbitrary linear differential operator with constant real coefficients were established in [10]. As far as almost trigonometric splines are concerned the result in [10] means that if $N>n$, then for every bounded $2 N$-periodic interpolated sequence, there exists a unique interpolating almost trigonometric spline.

In the present paper, we give another proof of this result and observe such an important feature that the inequality $N>n$ cannot be replaced by a weaker one (Theorem 1). After this, for $N>n$, we obtain a sharp estimate of the error of pointwise approximation by periodic interpolating almost trigonometric splines in the class of functions $W_{\infty}\left(\mathcal{L}_{2 n+2}\right)$ (Theorem 2).

Theorem 1. If $N>n$, then, for every bounded $2 N$-periodic sequence $\left\{y_{\nu}\right\}_{\nu \in \mathbb{Z}}, y_{\nu}=y_{\nu+2 N}$, there exists a unique $s \in S\left(\mathcal{L}_{2 n+2}, \Delta_{N}\right)$ such that $s(\nu h)=y_{\nu}, \nu \in \mathbb{Z}$.

If $N \leq n$, then periodic interpolating almost trigonometric spline cannot exist.

Let $N>n$. We set

$$
A_{n}(x)=\frac{x(x-h)}{4(n !)^{2}}+2 \sum_{\nu=1}^{n} \frac{(-1)^{\nu} \sin \frac{\nu x}{2} \sin \frac{\nu(x-h)}{2}}{\nu^{2}(n-\nu) !(n+\nu) ! \cos \frac{\nu h}{2}}
$$

for $0 \leq x \leq h$ and extend $A_{n}(x)$ to the whole real line by the equality $A_{n}(x+h)=-A_{n}(x)$ for $x \in \mathbb{R} \backslash[0, h]$.

We show that $A_{n} \in C^{(2 n+1)}(\mathbb{T})$. In the class $W_{\infty}\left(\mathcal{L}_{2 n+2}\right)$, the deviation from the periodic interpolating almost trigonometric splines is estimated by this function.

Theorem 2. If $N>n$, then, for every function $f \in W_{\infty}\left(\mathcal{L}_{2 n+2}\right)$, the inequality

$$
|f(x)-s(f)(x)| \leq 2\left|A_{n}(x)\right|
$$

holds at any point $x \in \mathbb{R}$. The inequality turns into an equality for $f(x)=2 A_{n}(x)$.

For interpolation by periodic polynomial splines, inequality (0.3) was proved by Tikhomirov [12]. For $N>3^{n-1} n$, inequality (0.3) is a particular case of the author's result [3]. For periodic trigonometric splines, the corresponding result was established by Nguen [5], [6, Ch. 2, §6].

\section{Auxiliary results}

First, we study the properties of the function $A_{n}(x)$.

Lemma 1. If $N>n$, then

$$
\left.A_{n}^{(j)}(x)\right|_{x=h}=-\left.A_{n}^{(j)}(x)\right|_{x=0}, \quad j=1,3, \ldots, 2 n+1,
$$

and

$$
\left.A_{n}^{(j)}(x)\right|_{x=h}=\left.A_{n}^{(j)}(x)\right|_{x=0}=0, \quad j=0,2, \ldots, 2 n .
$$


P r o o f. By easy calculations, we verify that $A_{n}(h)=A_{n}(0)=0$ and $\left.A_{n}^{\prime}(x)\right|_{x=h}=$ $-\left.A_{n}^{\prime}(x)\right|_{x=0}$. Further,

$$
\left.A_{n}^{\prime \prime}(x)\right|_{x=h}=\left.A_{n}^{\prime \prime}(x)\right|_{x=0}=\frac{1}{2(n !)^{2}}-\sum_{\nu=1}^{n} \frac{(-1)^{\nu-1}}{(n-\nu) !(n+\nu) !} .
$$

Using the known identity [7, Ch.IV, $\S 4.2 .1$, eq. 4], we obtain

$$
\sum_{\nu=1}^{n} \frac{(-1)^{\nu-1}}{(n-\nu) !(n+\nu) !}=\sum_{m=0}^{n-1} \frac{(-1)^{m+n-1}}{m !(2 n-m) !}=\frac{(-1)^{n-1}}{(2 n) !} \sum_{m=0}^{n-1}(-1)^{m}\left(\begin{array}{c}
2 n \\
m
\end{array}\right)=\frac{1}{2(n !)^{2}} \text {. }
$$

From this, it follows that $\left.A_{n}^{\prime \prime}(x)\right|_{x=h}=\left.A_{n}^{\prime \prime}(x)\right|_{x=0}=0$.

For $j=3,4, \ldots, 2 n+1$, we have

$$
A_{n}^{(j)}(x)=\sum_{\nu=1}^{n} \frac{(-1)^{\nu-1} \nu^{j-2} \cos (\nu(x-h / 2)+\pi j / 2)}{(n-\nu) !(n+\nu) ! \cos (\nu h / 2)} .
$$

For $j=2 k+1(k=1,2, \ldots, n)$, easy calculations yield

$$
\left.A_{n}^{(2 k+1)}(x)\right|_{x=h}=-\left.A_{n}^{(2 k+1)}(x)\right|_{x=0}=(-1)^{k} \sum_{\nu=1}^{n} \frac{(-1)^{\nu-1} \nu^{2 k-1} \tan (\nu h / 2)}{(n-\nu) !(n+\nu) !} .
$$

For $j=2 k(k=2,3, \ldots, n)$, we obtain

$$
\begin{gathered}
\left.A_{n}^{(2 k)}(x)\right|_{x=h}=\left.A_{n}^{(2 k)}(x)\right|_{x=0}=(-1)^{k} \sum_{\nu=1}^{n} \frac{(-1)^{\nu-1} \nu^{2 k-2}}{(n-\nu) !(n+\nu) !} \\
=\frac{(-1)^{n+k}}{(2 n) !} \sum_{m=0}^{n-1}(-1)^{m}(n-m)^{2 k-2}\left(\begin{array}{c}
2 n \\
m
\end{array}\right)=0 .
\end{gathered}
$$

Here, we used the identity [7, Ch.IV, $\S 4.2 .2$, eq. 34]. The lemma is proved.

We now extend the function $A_{n}(x)$ from $[0, h]$ to the whole real line by setting $A_{n}(x+h)=$ $-A_{n}(x)$. Lemma 1 gives that $A_{n}$ belongs to $C^{(2 n+1)}(\mathbb{R})$ and is $2 \pi$-periodic.

Lemma 2. If $N>n$, then $\mathcal{L}_{2 n+2}(D)\left(2 A_{n}(x)\right)=\operatorname{sign} \sin N x, x \in \mathbb{R}$.

P r o o f. Let $0 \leq x \leq h$. Since

$$
\sin \frac{\nu x}{2} \sin \frac{\nu(x-h)}{2}=A_{\nu} \cos \nu x+B_{\nu} \sin \nu x+C_{\nu}, \quad \nu=1,2, \ldots, n,
$$

where $A_{\nu}, B_{\nu}$ and $C_{\nu}$ are independent of $x$, the sum on the right-hand side of (0.2) vanishes by the differential operator $D\left(D^{2}+1^{2}\right)\left(D^{2}+2^{2}\right) \cdots\left(D^{2}+n^{2}\right)$. Taking into account that the factors on the right-hand side of $(0.1)$ can be rearranged, we obtain

$$
D^{2}\left(D^{2}+1^{2}\right) \cdots\left(D^{2}+n^{2}\right)\left(\frac{x(x-h)}{2(n !)^{2}}\right)=\frac{1}{2(n !)^{2}}\left(D^{2}+1^{2}\right) \cdots\left(D^{2}+n^{2}\right)(x(x-h))^{\prime \prime}=1 .
$$

For $x \in \mathbb{R} \backslash[0, h]$, we use the equality $A_{n}(x+h)=-A_{n}(x)$.

The next statement is a special case of a result proved in [9] for an arbitrary linear differential operator with constant real coefficients. 
Lemma 3. If $N>n$, then $x=0$ is the unique zero of $A_{n}(x)$ in $[0, h)$ and this zero is simple.

P r o o f. By Lemma $1, A_{n}(0)=0$. Moreover, the function $A_{n}(x)$ coincides, up to a nonzero constant, with some function $P_{n}(x)$ introduced in [9]. It was proved in [9] that if $N>n$, then $P_{n}(x)$ has a unique zero in $[0, h)$ and this zero is simple. Therefore, $A_{n}(x)$ has the same property.

To prove our two theorems, we also need the periodic analog of the Rolle theorem on the relation between the number of zeros of a smooth function $\varphi(x)$ and the number of sign changes of $D\left(D^{2}+1^{2}\right)\left(D^{2}+2^{2}\right) \cdots\left(D^{2}+n^{2}\right) \varphi(x)$ on $\mathbb{T}$.

We say that a continuous function $f$ changes sign at some point $t_{0}$ if the inequality $f\left(t_{0}-\varepsilon\right) f\left(t_{0}+\varepsilon\right)<0$ holds for all sufficiently small $\varepsilon>0$. If $f$ has a jump at the point $t_{0}$, then, instead of $f\left(t_{0}-\varepsilon\right)$ and $f\left(t_{0}+\varepsilon\right)$, we write $\lim _{t \rightarrow t_{0}-0} f(t)$ and $\lim _{t \rightarrow t_{0}+0} f(t)$, respectively. Denote by $Z(f, \mathbb{T})$ the number of zeros of the function $f$ on $\mathbb{T}$, and by $\nu(f, \mathbb{T})$ the number of sign changes of $f \not \equiv 0$ on $\mathbb{T}$ (the number of sign changes of $f \equiv 0$ is not defined). The number $\nu(f, \mathbb{T})$ is always even. We denote by $G(\mathbb{T})$ the set of $2 \pi$-functions of bounded variation with a finite number of jumps on the period and absolutely continuous on all intervals of continuity. We also denote by $G_{m}(\mathbb{T})$ the set of $2 \pi$-periodic functions whose derivatives of order $m-2$ are absolutely continuous on $\mathbb{T}$ and $f^{(m-1)} \in G(\mathbb{T})$. Let $\mathcal{T}_{n}$ be the set of trigonometric polynomials of order at most $n$.

Lemma 4. For every function $f \in G_{2 n+1}(\mathbb{T}) \backslash \mathcal{T}_{n}$, the following inequality holds:

$$
\nu\left(D\left(D^{2}+1^{2}\right)\left(D^{2}+2^{2}\right) \cdots\left(D^{2}+n^{2}\right) f, \mathbb{T}\right) \geq Z(f, \mathbb{T}) .
$$

This result was established by Nguen [5] (see also [6]) and is the periodic analog of the Rolle theorem for the trigonometric differential operator.

Note that the periodic analog of the Rolle theorem in the form of Lemma 4 exists not for any linear differential operator. More detailed information on some results and open problems in this area can be found in $[4]$ and references therein.

\section{Proofs of Theorems}

We now pass directly to the proofs of Theorems 1 and 2 .

P r o o f of Theorem 1. Let $N>n$. We prove that if $s \in S\left(\mathcal{L}_{2 n+2}, \Delta_{N}\right)$ and $s(j h)=0$ $\forall j \in \mathbb{Z}$, then $s \equiv 0$. After this, the existence and uniqueness of the interpolating periodic almost trigonometric spline for every interpolated periodic sequence is a simple consequence of the Kramer theorem for the corresponding system of linear algebraic equations.

Suppose that there exist $s_{1}, s_{2} \in S\left(\mathcal{L}_{2 n+2}, \Delta_{N}\right)$ such that $s_{k}(j h)=0 \forall j \in \mathbb{Z}(k=1,2)$ and $s_{1} \not \equiv s_{2}$. This means that there is a point $x_{*} \notin \Delta_{N}$ such that $s_{1}\left(x_{*}\right) \neq s_{2}\left(x_{*}\right)$. Let $s_{1}\left(x_{*}\right) \neq 0$ and $C=s_{2}\left(x_{*}\right) / s_{1}\left(x_{*}\right)$. Then the function $\varphi(x)=C s_{1}(x)-s_{2}(x)$ has the following properties:

1) $\varphi \in S\left(\mathcal{L}_{2 n+2}, \Delta_{N}\right)$;

2) $\varphi(j h)=0, \quad j=0,1, \ldots, 2 N-1$;

3) $\varphi\left(x_{*}\right)=0$.

Thus, $\varphi(x)$ has at least $2 N+1$ zeros on the period. From Lemma 4 , we have

$$
\nu\left(D\left(D^{2}+1^{2}\right)\left(D^{2}+2^{2}\right) \cdots\left(D^{2}+n^{2}\right) \varphi, \mathbb{T}\right) \geq 2 N+1 .
$$

But $D\left(D^{2}+1^{2}\right)\left(D^{2}+2^{2}\right) \cdots\left(D^{2}+n^{2}\right) \varphi(x)$ is a piecewise constant function with possible jumps at the points of the mesh $\Delta_{N}$. Therefore, this function cannot change sign more than $2 N$ times on $\mathbb{T}$. We have a contradiction from which it easily follows that $s_{1} \equiv s_{2} \equiv 0$. 
The inequality $N>n$ cannot be replaced by a weaker one. Indeed, if $N=n$, then the function $\sin n x$ interpolates the sequence $y \equiv 0$ at the points of $\Delta_{N}$. This function lies in the kernel of the linear differential operator $(0.1)$ and can be interpreted as an element of the space $S\left(\mathcal{L}_{2 n+2}, \Delta_{N}\right)$. Theorem 1 is proved.

P r o o f of Theorem 2 is based on the ideas of [12]. Let $N>n$. Suppose that (0.3) fails; i.e., there exist a point $x_{*} \in[0,2 \pi)$ and a function $f \in W_{\infty}\left(\mathcal{L}_{2 n+2}\right)$ such that the inequality

$$
\left|f\left(x_{*}\right)-s(f)\left(x_{*}\right)\right|>2\left|A_{n}\left(x_{*}\right)\right|
$$

holds. Define $\delta(x)=f(x)-s(f)(x)$. This function is zero at the points of the mesh $\Delta_{N}$. According to Lemma 3 , the function $A_{n}(x)$ vanishes at the same points. From these facts, we have $x_{*} \notin \Delta_{N}$. Therefore, there is a number $\lambda, 0<|\lambda|<1$, such that $\lambda \delta\left(x_{*}\right)=2 A_{n}\left(x_{*}\right)$.

We now introduce the function $\Delta(x)=\lambda \delta(x)-2 A_{n}(x)$. It is zero at all points of the set $\Delta_{N} \cup\left\{x_{*}\right\}$ and possibly also at some other points. Therefore $Z(\Delta(x), \mathbb{T}) \geq 2 N+1$. It is clear that $\Delta \in G_{2 n+1}(\mathbb{T}) \backslash \mathcal{T}_{n}$. We denote $\mathcal{L}_{2 n+1}(D)=D\left(D^{2}+1^{2}\right)\left(D^{2}+2^{2}\right) \cdots\left(D^{2}+n^{2}\right)$, apply Lemma 4 , and obtain

$$
\nu\left(\mathcal{L}_{2 n+1}(D) \Delta(x), \mathbb{T}\right) \geq 2 N+1 .
$$

From (0.1) and the definition of almost trigonometric splines, we have the equalities $\mathcal{L}_{2 n+1}(D) \delta(x)=$ $\mathcal{L}_{2 n+1}(D) f(x)-c_{j}$ on every interval $[j h,(j+1) h), j=0,1, \ldots, 2 N-1$, where $c_{j}$ are constants. Using the Lagrange finite increments formula and the inequality $|\lambda|<1$, we obtain

$$
\begin{aligned}
\mid \mathcal{L}_{2 n+1}(D)\left(\lambda \delta\left(t^{\prime}\right)\right) & -\mathcal{L}_{2 n+1}(D)\left(\lambda \delta\left(t^{\prime \prime}\right)\right)|<| \mathcal{L}_{2 n+1}(D) f\left(t^{\prime}\right)-\mathcal{L}_{2 n+1}(D) f\left(t^{\prime \prime}\right) \mid \\
& =\left|\mathcal{L}_{2 n+2}(D) f(\xi)\right| \cdot\left|t^{\prime}-t^{\prime \prime}\right| \leq\left|t^{\prime}-t^{\prime \prime}\right|
\end{aligned}
$$

on an arbitrary subinterval $\left[t^{\prime}, t^{\prime \prime}\right] \subset[j h,(j+1) h)$ for every interpolated function of our class. From $(0.2)$, it follows that $\mathcal{L}_{2 n+1}(D)\left(2 A_{n}(x)\right)=x-h / 2 \forall x \in[0, h)$. Hence, $\left|t^{\prime}-t^{\prime \prime}\right|=$ $\left|\mathcal{L}_{2 n+1}(D)\left(2 A_{n}\left(t^{\prime}\right)\right)-\mathcal{L}_{2 n+1}(D)\left(2 A_{n}\left(t^{\prime \prime}\right)\right)\right|$. Thus,

$$
\left|\mathcal{L}_{2 n+1}(D)\left(\lambda \delta\left(t^{\prime}\right)\right)-\mathcal{L}_{2 n+1}(D)\left(\lambda \delta\left(t^{\prime \prime}\right)\right)\right|<\left|\mathcal{L}_{2 n+1}(D)\left(2 A_{n}\left(t^{\prime}\right)\right)-\mathcal{L}_{2 n+1}(D)\left(2 A_{n}\left(t^{\prime \prime}\right)\right)\right| .
$$

It is easy to see that if $|a|<|b|$, then $\operatorname{sign}(b-a)=\operatorname{sign} b$. Applying this fact, we come to the conclusion that the function $\mathcal{L}_{2 n+1}(D) \Delta(x)$ changes sign no more than once in every interval $[j h,(j+1) h)$. If $\mathcal{L}_{2 n+1}(D) \Delta(x)$ changes sign at the point $j h$ (this is possible if the function is discontinuous at $j h)$, then $\mathcal{L}_{2 n+1}(D) \Delta(x)$ preserves sign in one of two adjacent intervals $((j-1) h, j h)$ or $(j h,(j+1) h)$. Thus, we arrive at the inequality

$$
\nu\left(\mathcal{L}_{2 n+1}(D) \Delta(x), \mathbb{T}\right) \leq 2 N .
$$

The obtained inequality contradicts to (2.1). The simple observation that inequality (0.3) turns into an equality for $f=2 A_{n}$ completes the proof.

Corollary 1. If $N>n$, then

$$
\sup _{f \in W \infty\left(\mathcal{L}_{2 n+2}\right)}\|f-s(f)\|_{p}=2\left\|A_{n}\right\|_{p}, \quad 1 \leq p<\infty,
$$

and

$$
\sup _{f \in W_{\infty}\left(\mathcal{L}_{2 n+2}\right)}\|f-s(f)\|_{\infty}=\left|\frac{h^{2}}{8(n !)^{2}}+4 \sum_{\nu=1}^{n} \frac{(-1)^{\nu} \sin ^{2} \frac{\nu h}{4}}{\nu^{2}(n-\nu) !(n+\nu) ! \cos \frac{\nu h}{2}}\right|
$$


We now consider separately the case $n=1$, i.e., the case of $\mathcal{L}$-splines corresponding to the differential operator $\mathcal{L}_{4}(D)=D^{2}\left(D^{2}+1\right)$. They belong piecewise to the space $\operatorname{span}\{1, t, \sin t, \cos t\}$. These splines generalize the well-known cubic splines and have many applications in numerical analysis for the shape preserving approximation, the description of curves and their parametrization, and other problems (see, for instance, [14], [15], [16], and references therein). In particular (see [15]) these splines are attractive from a geometrical point of view, because they are able to provide parameterizations of conic sections with respect to the arc length so that equally spaced points in the parameter domain correspond to equally spaced points on the described curve.

The restriction on the grid step is the least strong here: $h \leq \pi / 2$, and the "minimal" equidistant grid on the period is $\{0, \pi / 2, \pi, 3 \pi / 2\}$. Theorem 1 gives the existence and uniqueness of spline interpolants for $N \geq 2$. According to Corollary 1, the error of approximation in the class $W_{\infty}\left(\mathcal{L}_{4}\right)$ is

$$
\sup _{f \in W_{\infty}\left(\mathcal{L}_{4}\right)}\|f-s(f)\|_{\infty}=\left|1+\frac{\pi^{2}}{8 N^{2}}-\frac{1}{\cos \frac{\pi}{2 N}}\right| .
$$

\section{Conclusion}

We established that, for $2 \pi$-periodic $\mathcal{L}$-splines corresponding to the differential operator $(0.1)$ on the equidistant mesh with the step $h=\pi / N$, the restriction $N>n$ provides the existence and uniqueness of the $\mathcal{L}$-spline interpolant as well as the exact estimates of the error of approximation. This restriction is final, i.e., cannot be replaced by a weaker one.

\section{REFERENCES}

1. Korneichuk N.P. Splines in approximation theory. Moscow: Nauka, 1984. 352 p. [in Russian]

2. Micchelli C.A. Cardinal $L$-splines // Studies in spline functions and approximation theory, New York etc.: Acad. Press, 1976. P. 203-250.

3. Novikov S.I. Approximation of the class $W_{\infty}^{\mathcal{L}_{n}}$ by interpolation periodic $L$-splines // Approximation of functions by polynomials and splines, Sverdlovsk: Akad. Nauk SSSR, Ural. Sc. Center, 1985. P. 118-126. [in Russian]

4. Novikov S.I. Generalization of the Rolle theorem // East J. Approx., 1995. Vol. 1, no. 4. P. 571-575.

5. Nguen Thi T.H. The operator $D\left(D^{2}+1^{2}\right) \cdots\left(D^{2}+n^{2}\right)$ and trigonometric interpolation // Anal. Math., 1989. Vol. 15, no. 4. P. 291-306. [in Russian]

6. Nguen Thi T.H. Extremal problems for some classes of smooth periodic functions // Doctoral dissertation, 1994. Moscow: Steklov Institute of Math. 219 p. [in Russian]

7. Prudnikov A.P., Brychkov Yu.A., Marichev O.I. Integrals and series. Elementary functions, Moscow: Nauka, 1981. 800 p. [in Russian]

8. Schoenberg I.J. On Micchelli's theory of cardinal $L$-splines // Studies in spline functions and approximation theory, New York etc.: Acad. Press, 1976. P. 251-276.

9. Shevaldin V.T. A problem of extremal interpolation // Mat. Zametki, 1981. Vol. 29, no. 4. P. 603-622. [in Russian]

10. Shevaldin V.T. Interpolation periodic $L$-splines with uniform nodes // Approximation of functions by polynomials and splines, Sverdlovsk: Akad. Nauk SSSR, Ural. Sc. Center, 1985. P. 140-147. [in Russian]

11. Stechkin S.B., Subbotin Yu.N. Splines in numerical mathematics. Moscow: Nauka, 1976. 248 p. [in Russian]

12. Tikhomirov V.M. Best methods of approximation and interpolation of differentiable functions in the space $C[-1,1] / /$ Mat. Sb., 1969. Vol. 80, no. 122. P. 290-304. [in Russian]

13. Zhensykbaev A.A. Approximation of differentiable periodic functions by splines on a uniform subdivision // Mat. Zametki, 1973. Vol. 13, no. 6. P. 807-816. [in Russian]

14. Zhang J. $C$-curves: an extension of cubic curves // Comput. Aided Geom. Design, 1996. Vol. 13. P. 199-217. 
15. Roman F., Manni C., Speleers H. Spectral analysis of matrices in Galerkin methods based on generalized $B$-splines with high smoothness // Numer. Math., 2017. Vol. 135, no. 1. P. 169-216. DOI: 10.1007/s00211-016-0796-z

16. Mainar E., Peña J.M., Sánchez-Reyes J. Shape preserving alternatives to the rational Bezier model // Comput. Aided Geom. Design, 2001. Vol. 18. P. 37-60. DOI: 10.1016/S0167-8396(01)00011-5 